\title{
The Parabolic Path of a Projectile.
}

The proof, usually given, that the path of a projectile in vacuo is a parabola, assumes the equivalent of the equation to a parabola referred to a tangent and the diameter through the point of contact as axes.

The following proof ${ }^{*}$ requires only the theorem, $\mathrm{PN}^{2}=4 \mathrm{AS} . \mathrm{AN}$.

Let $A$ be the highest point of the path of the projectile.

Let $\mathbf{P}$ be the position of the projectile at time $t$ after the moment of projection; V cosa, Vsina the horizontal and vertical components of the initial velocity.

Let a vertical line through $A$ and a horizontal line through $P$ meet in $\mathrm{N}$.

Then

vertical component of velocity at time $t=\mathrm{V} \sin \alpha-g t$,

and time required to travel the path $\mathrm{AP}=\frac{\mathrm{Vsina}-g t}{g}$;

$$
\begin{aligned}
& \therefore \quad \mathrm{PN}=\frac{\mathrm{V} \cos \alpha(\mathrm{V} \sin \alpha-g t)}{g} \\
& \text { and } \quad \mathrm{AN}=\frac{(\mathrm{V} \sin \alpha-g t)^{2}}{2 g} ; \\
& \therefore \quad \mathrm{PN}^{2}=\frac{2 \mathrm{~V}^{2} \cos ^{2} \alpha}{g} \cdot \mathrm{AN} . \quad \therefore \text { etc. }
\end{aligned}
$$

\footnotetext{
* Given to me by J. G. Gibson, one of my pupils.-P.P.
} 